\title{
Metaloproteinases 1 e 7 e Câncer Colorretal
}

\author{
Metalloproteinases 1 and 7 and Colorectal Cancer
}

\section{MÁRIO JUCÁ'; ; BENICIO LUIZ BULHÕES BARROS PAULA NUNES ${ }^{2}$; HUNALDO LIMA DE MENEZES²; EDMUNDO GUILHERME DEALMEIDAGOMES ${ }^{3}$; DELCIOMATOS $^{4}$}

\author{
${ }^{1}$ Professor Associado de Coloproctologia da Faculdade de Medicina da Universidade Federal de Alagoas. \\ Coordenador da Disciplina de Coloproctologia da FAMED-UFAL; ${ }^{2}$ Aluno em Nivel de Doutorado da Universidade \\ Federal de São Paulo; ${ }^{3}$ Professor Assistente Mestre da Faculdade de Medicina da Universidade Federal de Alagoas; \\ ${ }^{4}$ Professor Titular da Escola Paulista de Medicina - Universidade Federal de São Paulo - Brasil.
}

JUCÁ M; NUNES BLBBP; MENEZES HL; GOMES EGA; MATOS D. Metaloproteinases 1 e 7 e Câncer Colorretal. Rev bras Coloproct, 2008;28(3): 353-362.

RESUMO: A metaloproteinase-1 (MMP-1) e a metaloproteinase-7 (MMP-7) são proteinases da matriz extracelular (MEC), zincodependentes, envolvidas no processo inicial da carcinogênese por permitirem a invasão tumoral na célula e promover o processo de metastatização. $O$ polimorfismo dessas proteinases tem sido estudado recentemente com o objetivo de validar susa expressão e/ou atividade como marcador prognóstico. Evidências cumulativas revelam importante papel das MMP's 1 e 7 em diferentes fases da carcinogênese. A MMP-1 tem ação direta sobre a principal proteína da MEC, que é o colágeno do tecido intersticial conectivo. Sua expressão aumentada neste tecido pode indicar alto potencial de disseminação tumoral em diferentes tipos de câncer, incluindo o colorretal. A associação deste aumento da expressão também parece ser verdadeira para a MMP-7.

Descritores: Neoplasia colorretal; Metalloproteinase 1; Metalloproteinase 7.

\section{INTRODUÇÃO}

A invasão celular de um tecido pode ocorrer durante vários estágios, sejam fisiológicos ou patológi$\cos$. Existem barreiras que se opõem ao movimento de invasão tecidual, como as membranas basais, a matriz estromal e a junção entre as células. Acredita-se que um mecanismo comum para a quebra destas barreiras, seja a atuação de enzimas proteolíticas. Os tipos de proteinases envolvidas dependem do tipo de tecido envolvido e das circunstâncias encontradas na matriz. Não obstante, existem três classes de proteinases (matriz metaloproteinases - MMP, serina proteinases e cisteína proteinases) que têm distribuição alterada, aumento na expressão e/ou atividade, envolvidas na remodelação da matriz, facilitando a invasão tumoral. Algumas evidências sugerem que proteinases, especi- ficamente as MMPs, estão envolvidas nos estados iniciais da progressão tumoral ${ }^{1}$.

A carcinogênese e o desenvolvimento do câncer colorretal são processos de várias etapas, caracterizadas por mudanças progressivas na quantidade ou atividade de proteínas que regulam a proliferação, diferenciação e sobrevida celular, e são mediados por mecanismos genéticos e epigenéticos ${ }^{2,3}$. Uma sequiência ordenada de eventos não aleatórios leva ao desenvolvimento do câncer colorretal (CCR), passando o epitélio por transformação invasiva ${ }^{4}$, sendo a progressão do epitélio intestinal normal até o desenvolvimento do carcinoma invasivo estimada entre 7 e 12 anos $^{5}$. A membrana basal e a matriz extracelular representam duas barreiras físicas à invasão maligna, e suas degradações pelas MMPs têm papel fundamental na progressão tumoral e disseminação das metástases ${ }^{4,6-11}$. 
Como os tumores primários necessitam crescer para se tornarem invasivos, a angiogênese é induzida como um dos principais mecanismos de sustentação da progressão tumoral ${ }^{12}$. Além disso, o desenvolvimento de uma microvasculatura funcional requer a organização tubular de células endoteliais, e sua maturação como veias estruturalmente estáveis e funcionalmente ajustáveis. A maturação dos vasos sangüíneos envolve o recrutamento de células murais, e deposição de uma matriz extracelular (MEC). Observações recentes sugerem que as MMPs podem ter papel importante na maturação da neovascularização tumoral ${ }^{13}$, ficando evidente que as MMPs participam dos mecanismos fisiológicos de desenvolvimento celular, e patológicos da carcinogênese ${ }^{12}$.

O tecido conectivo humano tem como proteína estrutural principal o colágeno, sendo os tipos I, II e III os mais abundantes, proporcionando a sustentação deste tecido, além de guiarem a migração, proliferação e diferenciação celular ${ }^{14}$. O colágeno intersticial é composto por 3 cadeias alfa de aproximadamente 1000 resíduos com repetidas cadeias trigêmeas Gly-X-Y, onde $\mathrm{X}$ e Y são prolina e hidroxiprolina, respectivamente, sendo esta conformação de tripla hélice o que faz o colágeno intersticial ser resistente à maioria das proteinases. As enzimas que podem clivar esta estrutura são conhecidas como colagenase ${ }^{15}$ e catepsina $\mathrm{K}$, esta última produzida pelos osteoclastos ${ }^{16}$. Enquanto a catepsina K quebra o colágeno I em um meio ácido especializado primariamente na reabsorção óssea, as demais enzimas colagenolíticas agem em $\mathrm{pH}$ neutro, e são membros da família das MMPs, sendo produzidas por diversos tecidos, incluindo as células estromais, epiteliais, macrófagos e leucócitos ${ }^{17}$.

\section{MATRIZES METALOPROTEINASES (MMPS)}

As MMPs são uma família de enzimas zincodependentes, intimamente relacionadas, que degradam a matriz extracelular, e são consideradas como importantes facilitadoras para a invasão e disseminação tumoral ${ }^{5-7,} 18-41$. Estas enzimas coletivamente podem degradar todos os componentes da matriz extracelular e são classificadas em grupos, incluindo colagenases, gelatinases, estromalisinas e metaloproteinases de membrana ${ }^{23,32,34,42}$. Podem ser divididas em dois grupos estruturalmente distintos: as secretadas e as de membrana ${ }^{43,44}$. As MMPs são secretadas como zimógenos inativos, e estudos in vitro demonstraram que o sistema u-PA/plasmin localizado na membrana celular, direta ou indiretamente, ativa um número de pro-MMPs, tais como pro-MMP-1, pro-MMP-3, proMMP-9, pro-MMP-10 e pro-MMP-13 ${ }^{45-47}$. Em adição à ativação zimogênica, a atividade da MMP é regulada pelos inibidores teciduais de metaloproteinases (IT-MMPs) ${ }^{48}$. As suas denominações são dadas a partir de sua atividade catalítica ${ }^{1}$. Existem membros da família das MMPs com atividade enzimática, virtualmente contra todos os componentes da MEC e membranas basais. Mais importante, a família das MMPs inclui as únicas enzimas capazes de clivar e desnaturar fibras do colágeno. As composições da MEC e membrana basal variam de acordo com a localização, mas geralmente incluem várias formas de colágeno, glicoproteínas (laminina, fibronectina, entactina, nidogênio), proteoglicanas e glicosaminoglicanas. A penetração e/ ou degradação da membrana basal ou MEC requer várias atividades proteolíticas diferentes, devido à diversidade de sua composição macromolecular. Por isso, durante a remodelação da matriz ocorre uma cascata complexa de eventos proteolíticos envolvendo múltiplos membros da família das MMPs ${ }^{1,79}$.

Os inibidores de tecido de metaloproteinases (IT-MMPs) são os principais inibidores fisiológicos das $\mathrm{MMPs}^{49-51}$. São proteínas secretadas que se une às MMPs individualmente, e regulam a atividade de cada MMP. Juntas, as MMPs e IT-MMPs formam um sistema biológico complexo que controla estritamente a degradação da MEC. As MMPs e IT-MMPs têm uma participação significativa na facilitação da invasão tumoral e metástases ${ }^{23,25,32,34,49,50}$, não apenas na degradação direta da MEC, mas também na interação com outros sistemas biológicos implicados na invasão tumoral, incluindo moléculas de adesão, proteínas citoesqueléticas e fatores de crescimento ${ }^{36,52,85}$.

A família das MMPs podem ser agrupadas e subdivididas de acordo com o número e características de domínios protéicos funcionais específicos. Com exceção da matrilisina, todas MMPs possuem um domínio hemopexina/vitronectina-like carboxiterminal que pode ter diferentes funções em cada um dos membros das MMPs. A região em "dobradiça", que liga os domínios hemopexina e catalítico, pode ser importante e determina a especificidade do substrato. Geralmente, esta região é variável na espessura e composição entre os membros da família, mas todas MMPs capazes de degradar fibras colágenas contém uma "dobradiça" 
de diferentes tamanhos e composições ${ }^{53}$. Em todos os membros da família MMP, o domínio catalítico contem três histidinas conservadas. A estrutura de toda a colagenase ativa confirma que, o zinco contido nos sítios catalíticos é coordenado por estas histidinas conservadas. Todas MMPs são sintetizadas como zimógenos inativos. Após a ativação, as MMPs são expostas à regulação por uma família de inibidores naturais de proteínas denominadas inibidores teciduais de metaloproteinases (IT-MMP), que ligam tanto as proMMPs ou MMPs ativas, inibindo a ativação autocatalítica de enzimas latentes, bem como a capacidade proteolítica de proteinases ativas. IT-MMPs podem ser inativadas proteoliticamente por diversas proteinases, como por exemplo a elastase neutrofílica (uma serina proteinase), tripisina e estromalisina- $3^{54}$. As MMPs também podem ser inibidas por associações não covalentes com a á2-macroglobulina ${ }^{21}$.

Vários estudos têm investigado MMPs e ITMMPs específicas no câncer colorretal, e vários outros estudos têm proposto significância prognóstica para MMPs individualmente (MMP- ${ }^{55}$, MMP- ${ }^{56}$, MMP$9^{57}$, IT-MMP1 ${ }^{58}$ e IT-MMP2 ${ }^{59}$ ) neste tipo de tumor. Entretanto, cada um destes estudos tem colocado o foco em uma única MMP ou IT-MMP, não existindo estudos maiores na expressão das MMPs e IT-MMPs no câncer colorretal. Estes achados diversos de MMPs e IT-MMPs individualmente em diferentes grupos de câncer colorretal, levaram CURRAN et al (2004) $)^{38}$ a realizarem um estudo compreensivo de MMPs em uma série bem caracterizada de câncer colorretal para se entender a interação entre as várias MMPs/IT-MMPs e compreender completamente a biologia das MMPs/ IT-MMPs no câncer colorretal. Neste estudo, foram analisados por imuno-histoquímica a expressão de todas as principais MMPs e IT-MMPs em um grupo bem caracterizado de câncer colorretal, sendo demonstrado que a composição molecular das MMPs/IT-MMPs identifica o grupo de pacientes com pior prognóstico.

\section{MATRIZ METALOPROTEINASE-1 E CÂNCER COLORRETAL}

A colagenase intersticial foi o primeiro membro identificado da família das MMPs ${ }^{60}$. Desde então, as MMPs estão relacionadas a mais de 25 tipos de enzimas zinco-dependentes que compartilham domínios funcionais comuns, tendo seus nomes descritivos associados a um substrato preferencial, e a um siste- ma de numeração dentro da família MMP, baseados em sua ordem de descobrimento ${ }^{5,40,61}$. Desta forma, a colagenase intersticial é também denominada de Metaloproteinease-1 (MMP-1) e degrada, especificamente, os colágenos tipo I, II e III ${ }^{28,62}$. Estas enzimas são codificadas por diferentes genes, e produzidas por uma variedade de tipos celulares, incluindo células epiteliais, mesenquimais, inflamatórias e neoplásicas ${ }^{63}$.

Uma colagenase típica é sintetizada como uma pré-próenzima e secretada, como uma pró-enzima inativa que consiste em um pró-peptídeo, um domínio catalítico, uma pequena região de ligação rica em prolina e um domínio hemopexina C-terminal (Hpx). A estrutura em forma de cristal da MMP-1 indica que seus domínios catalítico, e Hpx estão conectados com uma ligação peptídica ${ }^{64}$, mas o entendimento de como o domínio Hpx se comporta na quebra do colágeno é ilusório. Todavia, a base estrutural da especificidade da degradação do colágeno entre certos membros das MMPs, não está claramente compreendida. Um enigma adicional é o mecanismo pelo qual colagenases clivam a tripla hélice de colágeno, quando as dimensões do sítio ativo da colagenase e a estrutura do colágeno intersticial são consideradas ${ }^{65}$. O sítio de ligação do substrato da MMP-1 forma uma fenda profunda com o zinco localizado na porção inferior, e a entrada deste encaixe é de apenas $5 \AA$ de largura, o suficiente para acomodar apenas uma cadeia polipeptídica $^{14}$.

Várias linhas de evidência sugerem que as MMP's regulam o crescimento celular e a sobrevida, participando diretamente na geração de sinais que induzem à proliferação de células tumorais, ativando os precursores dos fatores de crescimento na superfície celular, liberando e ativando aqueles latentes seqüestrados na MEC e alterando a estrutura dos componentes essenciais da MEC ${ }^{5,40,66}$. A MMP-1 é uma típica enzima de membrana celular. Enquanto todas MMPs secretadas são normalmente liberadas como proenzimas, a MMP-1 pode ser liberada como enzima ativa antes de chegar à superfície celular ${ }^{63}$, tendo papel fundamental na remodelação da MEC, degradando diretamente vários de seus componentes e indiretamente ativando a pró-metaloproteinase- $2^{35}$. A presença deste tipo de MMP em espécimes de CCR tem sido associada com um pior prognóstico, independentemente do estadiamento de DUKES ${ }^{5,27,28,55,56,61,67}$. Entretanto, ROEB et al (2004) ${ }^{68}$ evidenciaram que a expressão da MMP-1 teve correlação significante com as 
metátases hematogênicas do CCR, mas nenhuma correlação com outros fatores clínico-patológicos, sugerindo que outros estudos são necessários para se ter uma melhor evidência deste aspecto, bem como de qual a origem deste aumento da expressão da MMP-1 no CCR. Este aumento da expressão da MMP-1 em pacientes com metástases de CCR, também foi verificado por HORIUCHI et al (2003) $)^{69}$.

BAKER et al $(2000)^{70}$ realizaram estudo no qual os níveis de MMP-1 no tumor colorretal foram maiores que no tecido normal, sendo ainda correlacionado significantemente com o estádio de Dukes, diferenciação tumoral, invasão linfática ( $<<0,05)$, acreditando que os níveis de MMP-1 parecem ser mais importantes na progressão do CCR, e o seguimento destes pacientes é necessário para determinar o quanto altos níveis de MMP-1 está associado com um pior prognóstico $27,33,52,55,71-73$. SARDINHA et al (2000) ${ }^{62}$ verificaram uma associação entre a expressão de MMP-1 e o aumento do estadiamento tumoral. Também no ano de 2000, SHIOZAWA et $\mathrm{al}^{27}$ encontraram imunorreatividade para MMP-1 em 76,1\% dos adenocarcinomas colorretais estudados (108 casos), sendo sua expressão menos freqüente e de menor intensidade nos carcinomas intramucosos do que nos invasivos ( $\mathrm{p}<0,0001)$. SUNAMI et al $(2000)^{28} \mathrm{en}-$ contraram uma correlação significante da expressão da MMP-1 nas metástases hematogênicas, porém não conseguiram correlação com outros achados clínicopatológicos. Em 2003, BENDARDAF et al $l^{35}$ verificaram níveis de expressão da MMP-1 significantemente acima da mediana, naqueles casos de tumores localizados no lado esquerdo do cólon, porém não houve associação significativa com o sexo, diferenciação histológica ou níveis de antígeno carcinoembriogênico. Porém, houve associação significante de baixos níveis com maior sobrevida $(\mathrm{p}=0,05)$. NOSHO et al $(2005)^{66}$ encontraram uma superexpressão de MMP-1 em $37,8 \%$ dos 90 tumores colorretais estudados, tendo uma correlação estatisticamente significante com o tamanho do tumor $(\mathrm{p}=0,0035)$.

\section{MATRIZ METALOPROTEINASE-7 E CÂNCER COLORRETAL}

A metaloproteinase 7 (MMP-7) faz parte da família das endopeptidases, com atividade hidrolítica de amplo espectro para as proteínas extracelular ${ }^{74}$. A MMP-7 é o menor membro das MMPs ${ }^{75-76}$. Compa- rando com as outras MMPs, a MMP-7 se distingue pelo seu baixo peso molecular (28 kDA) e ausência do domínio $\mathrm{C}$-terminal. Foi identificada primeiro em útero de ratas pós-parto ${ }^{77,78}$.

Apesar de ser classificada estrutural e funcionalmente como uma estromalisina, pertencendo ao mesmo grupo das MMP-3, -10, -11 e -26, percebe-se que há uma sobreposição entre as sub-classes, significando que as MMPs não têm necessariamente um substrato definido, sendo esta característica ainda mais marcante em processos patológicos, como a proteólise relacionada à invasão tumoral. Além disso, a ativação das mesmas pode envolver uma reação em cascata, visto que as atividades das MMPs interferem umas com as outras, através da ativação de proenzimas ${ }^{80}$.

A MMP-7 tem uma ampla atividade proteolítica, degradando vários componentes da membrana extracelular, entre eles os proteoglicanos, laminina, elastina, caseína, fibronectina e o colágeno tipo IV ${ }^{81-}$ 84. A degradação do colágeno tipo IV, em particular, torna a MMP-7 um importante fator de metástase, uma vez que o colágeno tipo IV é o maior componente da membrana basal. Uma característica importante da MMP-7 é a sua presença exclusivamente em tecido tumoral, não sendo encontrada em tecido colônico sadio. Outra característica que lhe torna peculiar, é que a sua secreção é feita unicamente por células de tecido epitelial $^{68,82}$.

A atividade das MMPs é regulada por expressão gênica, por ativação da pró-enzima, por inativação da enzima ativa e por inibidores tissulares específicos. As MMPs ampliam o processo de invasão tumoral, não só através da degradação das proteínas da matriz extra-celular, bem como pela ativação das cascatas que promovem a motilidade e a solubilização de fatores de crescimento ligadas à matriz extra-celular ${ }^{37}$. A expressão da MMP-7 é induzida por fatores de crescimento célula-célula e célula-matriz. O seu gene é induzível por estímulos extra-celulares que ativam um fator de transcrição dimérico, o complexo ativador de proteína 1 (AP-1), que vai se ligar ao promotor de MMPs no núcleo celular e ativar a sua transcrição. A indução da expressão e atividade da AP-1 são mediadas por três classes de proteína quinases ativadas por mitógenos (MAPK), ERKs, quinase do N-terminal de JUN ativada por estresse (JNK) e a de p38. A proteína quinase $\mathrm{C}$ (PKC) ativa a via de sinalização de ERK1/ERK2. Recentemente, tem-se mostrado que a super expressão de isoformas de PKC resulta na ativação do pro- 
motor de MMP- $1^{42}$. Por outro lado, alguns autores têm sugerido que a beta catenina é um importante fator regulador na super expressão da MMP- ${ }^{86}$. Estudos têm demonstrado que a troglitazona apresenta um efeito inibidor na síntese de MMP-7, por se ligar a um receptor hormonal expressado em tecido adiposo, nas adrenais e no baço, o receptor gama peroxisomal proliferador ativado (PPAR-g) que diminui a síntese de prostaglandinas ${ }^{87}$.

A MMP-7 tem sua expressão aumentada nas neoplasias de cabeça e pescoço, pulmão ${ }^{88}$; cólon $^{89}$, estômago ${ }^{90}$; pâncreas $^{91}$; mama $^{92}$.

A MMP-7 é a única das MMPs que só está expressa nos adenomas e neoplasias malignas, mas não em células colônicas normais. A contribuição da MMP7 no câncer colo-retal está bem estabelecida por manipulação genética da sua expressão no modelo experimental Min/+ (neoplasia intestinal múltipla) desenvolvido em ratos para câncer colorretal ${ }^{76,95}$. O nível da expressão da MMP-7 aumenta da seqüência adenoma para carcinoma e este nível de aumento se correlaciona com o estágio da doença e aparecimento de metástases, bem como um decréscimo na sobrevida. Análises multivariadas confirmam que a expressão da MMP-7 é um indicador significante de conseqüência de mortalidade ${ }^{96,97,98}$. Enquanto as MMP's classicamente têm sido implicadas na destruição da membrana basal, e associadas com estágios avançados do tumor e aparecimento de metástases, recentes estudos têm demonstrado que a MMP-7 está expressa em uma alta porcentagem de câncer colo-retal em fase inicial ${ }^{99}$. Estudos experimentais têm demonstrado que em ratos heterozigotos no modelo Min o RNA mensageiro foi induzido em $88 \%$ dos adenomas. Estes resultados sugerem que a MMP-7 pode contribuir para o desenvolvimento de tumor precoce ${ }^{95}$. Por outro lado, estudos clínicos têm mostrado que a MMP-7 tem a sua expressão aumentada em até $85 \%$ dos tumores colo-retais malignos ${ }^{95}$. Entretanto, outros fatores que regulam o gene de transcrição da MMP-7, desencadeando câncer colo-retal precoce ainda permanece sem elucidação ${ }^{83,100}$. De fato, com relação à MMP-7, tem se demonstrado tanto uma correlação positiva entre esta protease e potencial metastático quanto câncer colo-retal precoce. Um entendimento de como o gene da MMP-7 é regulado a nível transcricional é necessário para a determinação de como esta protease está super expressa em estágios precoces da tumorigênese do cólon. Examinando a sequiência de DNA do gene promotor, um maior discernimento pode ser obtido dentro dos fatores que regulam expressão do gene. A seqüência de análise da MMP-7 humana fomenta a revelação da presença de um número potencial de fator de transcrição, ligando elementos dentro da primeira base par no sítio do início da transcrição. Isto envolve o fator T-celular (TCF), ativador de proteína 1 (AP-1) e o sítio de ligação E-26 (ETS). A beta catenina tem mostrado interação com o sítio TCF, enquanto os fatores de transcrição AP-1 e ETS têm mostrado serem acionados por estímulos mitogênicos ${ }^{69,98,100}$.

Existem duas teorias para o crescimento do câncer colo-retal invasivo e metástase em pacientes com super expressão da MMP-7: uma é devido ao efeito direto da MMP-7, pelo fato de ela exibir um amplo espectro de especificidade e efetividade na degradação de vários componentes da membrana basal. A outra aponta para os efeitos indiretos da MMP-7, com a ativação das gelatinases A e B. Estudos preliminares mostram não haver diferença no grau de atividade das gelatinases nos tumores, sugerindo que a atividade da MMP-7 no câncer colo-retal é por efeito direto. Entretanto, outros efeitos indiretos da enzima não podem ser demonstrados, como a ativação de outras MMPs ou inativação de inibidores das proteinases ${ }^{77,101}$.

O papel da MMP-7 nas metástases foi testado diretamente, utilizando-se antisenso oligonucleotídeo em ratos com câncer colo-retal e metástase hepática, o que causou a inibição desta protease, diminuindo a invasão da membrana basal pela enzima, e inibindo a metástase hepática. Este resultado demonstra que a MMP-7 tem um importante papel nas metástases, e estágios avançados do câncer colo-retal. Estudos recentes in vitro demonstram que a presença do antisenso oligonucleotídeo inibe a MMP-7, prevenindo a invasão do cólon e metástase em até $50 \%{ }^{75,102-104}$.

Intestinos de pacientes com doença inflamatória intestinal apresentam risco de desenvolver câncer colo-retal e exibem níveis aumentados de MMP-1, -3, -7 e -14. A MMP-7 é a única protease que está aumentada em adenomas, comparando com tecido intestinal normal. A MMP-7 também se encontra expressada focalmente em $50 \%$ dos adenomas colônicos benignos, e super expressa em $90 \%$ dos adenomas de pacientes com polipose adenomatosa familiar ${ }^{75}$.

A MMP-7 é também sintetizada no endotélio vascular adjacente ao tumor, mas não em tecidos sadios. Em adição, estudos demonstram que cultura de células endoteliais de veia umbilical humana secreta 
MMP-7. Este resultado sugere a possibilidade de esta enzima estar envolvida na angiogênese do tumor ${ }^{77,78}$, 105 .

\section{CONSIDERAÇÕES FINAIS}

Matrilisina é uma das metaloproteinases que tem um papel crítico na invasão tumoral e na remodelagem da matrix extracelular no câncer colorretal. Muito embora, nenhuma metaloproteinase sozinha ou mesmo suas associações possam justifi- car o processo de desorganização pluricelular, que culmina com uma transcrição de mRNA, levando à transformação maligna de uma célula. O entendimento desses mecanismo certamente em um determinado momento, junto com a diversidade de conhecimentos que estão sendo desenvolvidos, trarão um caminho para a prevenção e algumas vezes o prognóstico de indivíduos onde a expressão desses marcadores possam ser decodificadas para uma estratificação clínica.

ABSTRACT: The metalloproteinase-1 (MMP-1) and metalloproteinase-7 (MMP-7) are proteinases of the extracellular matrix (MEC), zinc-dependent, involved in the initial process of carcinogenesis, allowing the invasion by the tumor cell and promoting the process of metastasis. The polymorphism of these proteinases has been studied recently in order to validate its expression and / or activity as a marker prognosis. Evidence shows cumulative important role of MMPs 1 and 7 in different stages of carcinogenesis. The MMP-1 is direct action on the main protein of the MEC, which is the collagen of interstitial connective tissue. Its increased expression in this tissue may indicate high potential for spread in different tumor types of cancer, including colorectal. The association of this increase of expression also appears to be true for MMP-7.

Key words: Colorectal neoplasms; Matrix metalloproteinase 1; Matrix metalloproteinase 7.

\section{REFERÊNCIAS}

1. Coussens LM, Werb Z - Matrix metalloproteinases and the development of cancer. Chem Biol 1996; 3(11): 895-904.

2. Wu AW, Gu J, Ji JF, Li ZF, Xu GW. Role of COX-2 in carcinogenesis of colorectal cancer and its relationship with tumor biological characteristics and patients' prognosis. World J Gastroenterol 2003; 9(9): 1990-1994.

3. Xiong B, Sun TJ, Hu WD, Cheng FL, Mao M, Zhou YF Expression of cyclooxygenase-2 in colorectal cancer and its clinical significance. World J Gastroenterol 2005; 11(8): 11051108 .

4. Ghilardi G, Biondi ML, Mangoni J, Leviti S, Demonti M, Guagnellini E et al - Matrix metalloproteinase-1 promoter polymorphism $1 \mathrm{G} / 2 \mathrm{G}$ is correlated with colorectal cancer invasiveness. Clin Cancer Res 2001; 7(8): 2344-2346.

5. Wagenaar-Miller RA, Gorden L, Matrisian LM - Matrix metalloproteinases in colorectal cancer: is it worth talking about? Cancer Metastasis Rev 2004; 23(1-2): 119-135.

6. Murphy G, Koklitis P, Carne AF - Dissociation of tissue inhibitor of metalloproteinases (TIMP) from enzyme complexes yields fully active inhibitor. Biochem J 1989; 261(3): 1031-1034.

7. Matrisian LM - Metalloproteinases and their inhibitors in matrix remodeling. Trends genet 1990; 6(4): 121-125.

8. Liotta LA, Stetler-Stevenson WG, Steeg PS - Cancer invasion and metastasis: positive and negative regulatory elements. Cancer Invest 1991; 9(5): 543-51.
9. Joo YE, Seo KS, Kim J, Kim HS, Rew JS, Park CS et al - Role of tissue inhibitors of metalloproteinases (TIMPs) in colorectal carcinoma. J Korean Med Sci 1999; 14(4): 417-423.

10. Stetler-Stevenson WG, Yu AE - Proteases in invasion: matrix metalloproteinases. Semin Cancer Biol 2001; 11(2): 143-52.

11. Moilanen M, Sorsa T, Stenman M, Nyberg P, Lindy O, Vesterinen $\mathrm{J}$ et al - Tumor-associated trypsinogen-2 (trypsinogen-2) activates procollagenases (MMP-1, -8, -13) and stromelysis-1 (MMP-3) and degrades type I collagen. Biochemistry 2003; 42(18): 5414-5420.

12. Deryugina EI, Quigley JP - Matrix metalloproteinases and tumor metastasis. Cancer Metastasis Rev 2006; 25(1): 9-34.

13. Chantrain CF, Henriet P, Jodele S, Emonard H, Feron O, Courtoy PJ et al - Mechanisms of pericyte recruitment in tumour angiogenesis: A new role for metalloproteinases. Eur J Cancer 2006; 42(3): 310-318.

14. Chung L, Dinakarpandian D, Yoshida N, Lauer-Fields JL, Fields GB, Visse R et al - Collagenase unwinds triple-helical collagen prior to peptide bond hydrolysis. EMBO Journal 2004; 23(15), 3020-3030.

15. Visse R, Nagase H - Matrix metalloproteinases and tissue inhibitors of metalloproteinases: structure, function, and biochemistry. Circ Res 2003; 92(8): 827-839.

16. Garnero P, Borel O, Byrjalsen I, Ferreras M, Drake FH, Mcqueney MS et al - The collagenolytic activity of cathepsin $\mathrm{K}$ is unique among mammalian proteinases. J Biol Chem 1998; 273(48): 32347-32352.

17. Sternlicht MD, Werb Z-How matrix metalloproteinases regulate cell behavior. Annu Rev Cell Dev Biol 2001; 17: 463-516. 
18. Liotta LA - Tumor invasion and metastases - role of the extracellular matrix. Cancer Res 1986; 46(1): 1-7.

19. Mignatti P, Rifkin DB - Biology and biochemistry of proteinases in tumor invasion. Physiol Rev 1993; 73(1): 161-195.

20. Stetler-Stevenson WG, Aznavoorian S, Liotta LA - Tumor cell interactions with the extracellular matrix during invasion and metastasis. Annu Rev Cell Biol 1993; 9: 541-573.

21. Birkedal-Hansen $\mathrm{H}$ - Proteolytic remodeling of extracellular matrix. Cur Opin Cell Biol 1995; 7(5): 728-735.

22. Chambers AF, Matrisian LM - Changing views of the role of matrix metalloproteinases in metastasis. J Natl Cancer Inst 1997; 89(17): 1260-1270.

23. Curran S, Murray GI - Matrix metalloproteinases in tumour invasion and metastasis. J Pathol 1999; 189(3): 300-308.

24. Kähäri VM, Saarialho-Kere U - Matrix metalloproteinases and their inhibitors in tumour growth and invasion. Ann Med 1999; 31(1): 34-45.

25. Curran S, Murray GI - Matrix metalloproteinases: molecular aspects of their roles in tumour invasion and metastasis. Eur J Cancer 2000; 36(13): 1621-1630.

26. Nelson AR, Fingleton B, Rothenberg ML, Matrisian LM Matrix metalloproteinases: biologic activity and clinical implications. J Clin Oncol 2000; 18(5): 1135-1149.

27. Shiozawa J, Ito M, Nakayama T, Nakashima M, Kohno S, Sekine I - Expression of matrix metalloproteinase-1 in human colorectal carcinoma. Mod Pathol 2000; 13(9): 925-933.

28. Sunami E, Tsuno N, Osada T, Saito S, Kitayama J, Tomozawa $\mathrm{S}$ et al - MMP-1 is a prognostic marker for hematogenous metastasis of colorectal cancer. Oncologist 2000; 5(2): 108114.

29. Stetler-Stevenson WG - The role of matrix metalloproteinases in tumor invasion, metastasis, and angiogenesis. Surg Oncol Clin N Am 2001; 10(2): 383-392.

30. Stetler-Stevenson WG, Yu AE - Proteases in invasion: matrix metalloproteinases. Semin Cancer Biol 2001; 11(2): 143-152.

31. Yamauchi K, Ogata Y, Nagase H, Shirouzu K - Inibition of liver metastasis from orthotopically implanted colon cancer in nude mice by transfection of the TIMP-1 gene into KM12SM cells. Surg Today 2001; 31(9): 791-798.

32. Brinckerhoff CE, Matrisian LM - Matrix metalloproteinases: a tail of a frog that became a prince. Nat Rev Mol Cell Biol 2002; 3(3): 207-214.

33. Overall CM, López-Otín C - Strategies for MMP inhibition in cancer: innovations for the post-trial era. Nat Rev Cancer 2002; 2(9): 657-672.

34. Yana I, Seiki M - MT-MMPs play pivotal roles in cancer dissemination. Clin Exp Metastasis 2002; 19(3): 209-215.

35. Bendardaf R, Lamlum H, Vihinen P, Ristamaki R, Laine J, Pyrhonen S - Low collagenase-1 (MMP-1) and MT1-MMP expression levels are favourable survival markers in advanced colorectal carcinoma. Oncology 2003; 65(4): 337-346.

36. Leeman MF, Curran S, Murray GI - New insights into the roles of matrix metalloproteinases in colorectal cancer development and progression. J Pathol 2003; 201(4): 528534.

37. Stamenkovic I - Extracellular matrix remodelling: the role of matrix metalloproteinases. J Pathol 2003; 200(4): 448-464.

38. Curran S, Dundas SR, Buxton J, Leeman MF, Ramsay R, Murray GI - Matrix metalloproteinase/tissue inhibitors of matrix metalloproteinase phenotype identifies poor prognosis colorectal cancers. Clin Cancer Res 2004; 10(24): 8229-8234.

39. Mariani SM - Conference report-Extracellular matrix and cancer: revisiting metalloproteinases highlights from the annual meeting of the American Society for Cell Biology; December 13-17, 2003; San Francisco, California. MedGenMed 2004; 6(1): 25 .

40. Zucker S, Vacirca J - Role of matrix metalloproteinases (MMPs) in colorectal cancer. Cancer Metastasis Rev 2004; 23(1-2): 101-117.

41. Tauro JR, Gemeinhart RA - Matrix metalloprotease triggered delivery of cancer chemotherapeutics from hydrogel matrixes. Bioconjug Chem 2005; 16(5): 1133-1139.

42. Westermarck J, Kähäri VM - Regulation of matrix metalloproteinase expression in tumor invasion. FASEB J 1999; 13(8): 781-792.

43. Collen A, Hanemaaijer R, Lupu F, Quax PHA, Van Lent N, Grimbergen J et al - Membrane-type matrix metalloproteinasemediated angiogenesis in a fibrin-collagen matrix. Blood 2003; 101(5): 1810-1817.

44. Maskos K, Bode W - Structural basis of matrix metalloproteinases and tissue inhibitors of metalloproteinases. Mol Biotechnol 2003; 25(3): 241-266.

45. Eeckhout Y, Vaes G - Further studies on the activation of procollagenase, the latent precursor of bone collagenase. Effects of lysosomal cathepsin B, plasmin and kallikrein, and spontaneous activation. Biochem J 1977; 166(1): 21-31.

46. Mazzieri R, Masiero L, Zanetta L, Monea S, Onisto M, Garbisa $S$ et al - Control of type IV collagenase activity by components of the urokinase-plasmin system: a regulatory mechanism with cell-bound reactants . EMBO J 1997; 16(9): 2319-2332.

47. Deryugina EI, Ratnikov BI, Yu Q, Baciu PC, Rozanov DV, Strongin AY - Prointegrin maturation follows rapid trafficking and processing of MT1-MMP in furin-negative colon carcinoma LoVo cells. Traffic 2004; 5(8): 627-641.

48. Woessner JF - Matrix metalloproteinases and their inhibitors in connective tissue remodeling. FASEB J 1991; 5(8): 21452154.

49. Jiang Y, Goldberg ID, Shi YE - Complex roles of tissue inhibitors of metalloproteinases in cancer. Oncogene 2002; 21(14): 2245-2252.

50. Baker AH, Edwards DR, Murphy G - Metalloproteinase inhibitors: biological actions and therapeutic opportunities. J Cell Sci 2002; 115(19): 3719-3727.

51. Holten-Andersen MN, Hansen U, Brunner N, Nielsen HJ, Illemann M, Nielsen BS - Localization of tissue inhibitor 
of metalloproteinases 1 (TIMP-1) in human colorectal adenoma and adenocarcinoma. Int J Cancer 2005; 113(2): 198-206.

52. Egeblad M, Werb Z - New functions for the matrix metalloproteinases in cancer progression. Nat Rev Cancer 2002; 2(3): 161-174.

53. Takino T, Sato H, Shinagawa A, Seiki M - Identification of the second membrane-type matrix metalloproteinase (MT-MMP2) gene from a human placenta cDNA library. MT-MMPs form a unique membrane-type subclass in the MMP family. $\mathrm{J}$ Biol Chem 1995; 270(39): 23013-23020.

54. Itoh $\mathrm{Y}$, Nagase $\mathrm{H}$ - Preferential inactivation of tissue inhibitor of metalloproteinases-1 that is bound to the precursor of matrix metalloproteinase 9 (progelatinase B) by human neutrophil elastase. J Biol Chem 1995; 270(28): 16518-16521.

55. Murray GY, Duncan ME, O’Neil P, Melvin WT, Fothergill JE - Matrix metalloproteinase-1 is associated with poor prognosis in colorectal cancer. Nat Med 1996; 2(4): 461-462.

56. Adachi Y, Yamamoto H, Itoh F, Arimura Y, Nishi M, Endo T et al - Clinicopathologic and prognostic significance of matrilysin expression at the invasive front in human colorectal cancers. Int J Cancer 2001; 95(5): 290-294.

57. Zeng ZS, Huang Y, Cohen AM, Guillem JG - Prediction of colorectal cancer relapse and survival via tissue RNA levels of matrix metalloproteinase-9. J Clin Oncol 1996; 14(12): 31333140.

58. Zeng ZS, Cohen AM, Zhang ZF, Stetler-Stevenson W, Guillem $\mathrm{JG}$ - Elevated tissue inhibitor of metalloproteinase 1 RNA in colorectal cancer stroma correlates with lymph node and distant metastases. Clin Cancer Res 1995; 1(8): 899-906.

59. Ring P, Johansson K, Höyhtyä M, Rubin K, Lindmark G Expression of tissue inhibitor of metalloproteinases TIMP-2 in human colorectal cancer - a predictor of tumour stage. Br J Cancer 1997; 76(6): 805-811.

60. Gross J, Lapiere CM - Collagenolytic activity in anphibian tissues; a tissue culture assay. Proc Natl Acad Sci USA 1962; 48(6): 1014-1022.

61. Zinzindohoue F, Lecomte T, Ferraz JM, Houllier AM, Cugnenc $\mathrm{PH}$, Berger A et al - Prognostic significance of MMP-1 and MMP-3 functional promoter polymorphisms in colorectal cancer. Clin Cancer Res 2005; 11(2-1): 594-599.

62. Sardinha TC, Nogueras JJ, Xiong H, Weiss EG, Wexner SD, Abramson S - Membrane-Type 1 matrix metalloproteinase mRNA expression in colorectal cancer. Dis Colon Rectum 2000; 43(3): 389-395.

63. Murai S, Umemiya T, Seiki M, Harigaya K - Expression and localization of membrane-type-1 matrix metalloproteinase, $\mathrm{CD}$ 44, and laminin-5gamma2 chain during colorectal carcinoma tumor progression. Virchows Arch 2004; 445(3): 271-278.

64. Li J, Brick P, O'Hare MC, Skarzynski T, Lloyd LF, Curry VA et al - Structure of full-length porcine synovial collagenase reveals a C-terminal domain containing a calcium-linked, fourbladed beta-propeller. Structure 1995; 3(6): 541-549.
65. Bode W - A helping hand for collagenases: the haemopexinlike domain. Structure 1995; 3(6): 527-530.

66. Nosho K, Yoshiday M, Yamamoto H, Taniguchi H, Adachi Y, Mikami $\mathrm{M}$ et al - Association of Ets-related transcriptional factor E1 AF expression with overexpression of matrix metalloproteinases, COX-2 and iNOS in the early stage of colorectal carcinogenesis. Carcinogenesis 2005; 26(5): 892-899.

67. Van Der Stappen JW; Hendriks T; Wobbes T - Correlation between collagenolytic activity and grade of histological differentiation in colorectal tumors. Int J Cancer 1990; 45(6): 1071-1078.

68. Roeb E, Arndt M, Jansen B, Schumpelick V, Matern S Simultaneous determination of matrix metalloproteinase (MMP)-7, MMP-1, -3, and -13 gene expression by multiplex PCR in colorectal carcinomas. Int J Colorectal Dis 2004; 19(6): 518-524.

69. Horiuchi S, Yamamoto H, Min Y, Adachi Y, Itoh F, Imai K Association of ets-related transcriptional factor E1AF expression with tumour progression and overexpression of MMP-1 and matrilysin in human colorectal cancer. J Pathol 2003; 200(5): 568-576.

70. Baker EA, Bergin FG, Leaper DJ - Matrix metalloproteinases, their tissue inhibitors and colorectal cancer staging. Br J Surg 2000; 87(9): 1215-21.

71. Hotary K, Allen E, Punturieri A, Yana I, Weiss SJ - Regulation of cell invasion and morphogenesis in a three-dimensional type I collagen matrix by membrane-type matrix metalloproteinases 1, 2, and 3. J Cell Biol 2000; 149(6): 1309-1323.

72. Hotary KB, Yana I, Sabeh F, Li XY, Holmbeck K, BirkedalHansen $\mathrm{H}$ et al - Matrix metalloproteinases (MMPs) regulate fibrin-invasive activity via MT1-MMP-dependent and independent processes. J Exp Med 2002; 195(3): 295-308.

73. López-Otín C, Overall CM - Protease degradomics: a new challenge for proteomics. Nat Rev Mol Cell Biol 2002; 3(7): 509-519.

74. Bertini I, Calderone V, Cosenza M. Conformational variability of matrix metalloproteinases: Beyond a single 3D structure. PNAS 2005; 102 (15): 5334-5339.

75. Hasegawa S, Koshikawa N, Momiyama N, Moriyama K, Miyazaki K, Ichikawa Y, Ichikawa T, Mitsuhashi M, Shimada H, Miyazaki K. Matrilysin-specific antisense oligonucleotide inhibits liver metastasis of human colon cancer in a nude mouse model. Int J Cancer 1998; 76(6): 812-816.

76. Kioi M, Yamamoto K, Higashi S, Koshikawa N, Fijita K, Miyazaki K. matrilysin (MMP-7) induces homotypic adhesion of human colon cancer cells and enhances their metastatic potential nude mouse model. Oncogene 2003; 22(54): 8662-8670.

77. Adachi Y, Yamamoto H, Itoh F, Hinoda Y, Okada Y, Imai K. Contribution of matrilysin (MMP-7) to the metastatic pathway of human colorectal cancers. Gut 1999; 45(2): 252-258.

78. Nishizuka I, Ichikawa Y, Ishikawa T, Kamiyama M, Hasegawa S, Momiyama N, Miyazaki K, Shimada H. Matrilysin 
stimulates DNA synthesis of cultured vascular endothelial cells and induces angiogenesis in vivo. Cancer Letters 2001; 173(2): 175-182.

79. Cawston, TE . Fragments of human fibroblast collagenase. Purification and characterization. Biochem J. 1989 October 1; 263(1): 201-206.

80. Saha D, Roman C, Beauchamp D. New strategies for colorectal cancer prevention and treatment. World J Surg 2002; 26(7): 762-766.

81. Wilson CL, Matrisian LM. Matrilysin: An epithelial matrix metalloproteinase with potentially novel functions. Int $\mathbf{J}$ Biochem Cell Biol 1996; 28(2): 123-136.

82. Ichikawa Y, Ishikawa T, Momiyama N, Yamaguchi S, Masui $\mathrm{H}$, Hasegawa $\mathrm{S}$ et al. Detection of regional lymph node metastase in colon cancer using RT-PCR for matrix metalloproteinase 7, matrilysin. Clin Exp Metastasis 1998; 16(1): 3-8.

83. Masaki T, Matsuoka H, Sugiyama M, Abe N, Goto A, Sakamoto A, Atomi Y. Matrilysin (MMP-7) as a significant determinant of malignant potential of early invasive colorectal carcinomas. Br J Cancer 2001; 84(10):1317-321.

84. Yanagisawa N, Geironson L, Al-Soud WA, Ljungh A. Expression of matrix metalloproteinase-2, -7 and -9 on human colon, liver and bile duct cell lines by enteric nd gastric helicobacter species. FEMS Immunol Med Microbiol 2005; 44(2): p197-204.

85. McCawley LJ, Matrisian LM. Matrix metalloproteinases: they're not just for matrix anymore. Curr Opin Cell Biol. 200; 13(5):534-40.

86. Brabletz T, Jung A, Dag S, Hlubek F, Kirchner T. Beta-catenin regulates the expression of the matrix metalloproteinase-7 in human colorectal cancer. Am J Pathol 1999; 155(4): 10331038 .

87. Sunami E, Tsuno NH, Kitayama J, Saito S, Osada T, Yamagushi H, Tomozawa S, Tsuruo T, Shibata Y, Nagawa H. Decreased synthesis of matrix metalloproteinase- 7 and adhesion to the extracellular matrix proteins of human colon cancer cells treated with troglitazone. Surg Today 2002; 32(4): 343-350.

88. Muller D, Breathnach R, Engelmann A, Millon R, Bronner G, Flesch $\mathrm{H}$ et al. Expression of collagenase-related metalloproteinase genes in human lung or head and neck tumours. Int J Cancer 1991; 48(4): 550-556.

89. Yamamoto H, Itoh F, Hinoda Y, Senota A, Yoshimoto M, Nakamura $\mathrm{H}$ et al. Expression of matrilysin mRNA in colorectal adenomas and its induction by truncated fibronectin. Biochem Biophys Res Commun 1994; 201(2): 657-664.

90. McDonnell S, Navre M, Coffey RJ Jr, Matrisian LM. Expression and localization of the matrix metalloproteinase pump-1 (MMP-7) in human gastric and colon carcinomas. Mol Carcinog 1991; 4(6): p527-533.

91. Bramhall SR, Neoptolemos JP, Stamp GW, Lemoine NR. Imbalance of expression of matrix metalloproteinases (MMPs) and tissue inhibitors of the matrix metalloproteinases (TIMPs) in human pancreatic carcinoma. J Pathol 1997; 182(3): 347355 .

92. Basset P, Bellocq JP, Wolf C, Stoll I, Hutin P, Limacher JM, Podhajcer OL, Chenard MP, Rio MC, Chambon P. A novel metalloproteinase gene specifically expressed in stromal cells of breast carcinomas. Nature 1990; 348(6303): 699-704.

93. Roeb E, Matern S. Matrix metalloproteinases: Promoters of tumor invasion and metastasis - A review with focus on gastrointestinal tumors. Z Gastroenterol. 200; 39(9):807-13.

94. Lew RA. The zinc metallopeptidase family: new faces, new functions. Protein Pept Lett 2004; 11(5): 407-414.

95. Wilson CL, Heppner KJ, Labosky PA, Hogan BLM, Matrisian LM. Intestinal tumorigenesis is suppressed in mice lacking the metalloproteinase matrilysin. Proc Natl Acad Sci USA 1997; 94(4): 1402-1407.

96. Ougolkov AV, Yamashita K, Mai M, Minamoto T. Oncogenic b-catenin and MMP-7 (matrilysin) congregate in late-stage clinical colon cancer. Gastroenterology 2002; 122(1):60-71.

97. Yamamoto H, Iku S, Adachi Y, Imsumran A, Taniguchi H, Nosho K, Min Y, Horiuchi S, Yoshida M, Itoh F, Imai K. Association of trypsin expression with tunour progression and matrilysin expression in human colorectal cancer. J Pathol 2003;199(2): 176-184.

98. Husoy T, Olstorn HB, Knutsen HK, Loberg EM, Cruciani V, Mikalsen SO, Goverud IL, Alexander J. Truncated mouse adenomatous polyposis coli reduces connexin 32 content and increases matrilysin secretion from Paneth cells. Eur J Cancer 2004; 40(10): 1599-1603.

99. Lloyd JM, McIver CM, Stephenson SA, Hewett P, Rieger N, Hardingham JE. Identification of early-stage colorectal cancer patients at risk fo relapse post-ressection by immunobead reverse transcription-PCR analysis of peritoneal lavage fluid for malignant cells. Clin Cancer Res 2006; 12(2): 417-423.

100. Lynch C, Crawford HC, Matrisian LM, McDonnell S. Epidermal growth factor upregulates matrix metallopreoteinase-7 expression trough activation of PEA3 transcription factors. Int J Oncol 2004; 24(6): 1565-1572.

101. Seiler N, Schneider Y, Gosse F, Schleiffer R, Raul F. Polyploidisation of metastatic colon carcinoma cells by microtubule and tubulin interacting drugs: effect on proteolytic activity and invasiveness. Int J Oncol 2004; 25(4): 1039-1048.

102. Miyazaki K, Koshikawa N, Hasegawa S, Momiyama N, Nagashima Y, Moriyama K, Ichikawa Y, Ichikawa T, Mitsuhashi M, Shimada H. Matrilysin as a target for chemotherapy for colon cancer: use of antisense oligonucleotides as antimetastatic agents. Cancer Chemother Pharmacol 1999; 43(Suppl): S52-S55.

103. Kumar A, Collins H, Van Tan J, Scholefield JH, Watson AS. Effect of preoperative radiotherapy on matrilisyn gene expression in rectal cancer. Eur J Cancer 2002; 38(4): p505-510.

104. Kurokawa S, Arimura Y, Yamamoto H, Adachi Y, Endo T, Sato T, Suga T, Hosokawa M, Shinomura Y, Imai K. Tumor 
matrilysin expression predctis metastatic potential of stage I (pT1) colon and rectal cancers. Gut 2005; 54(12): 1751-1758.

105. Takahashi Y, Ellis LM, Mai M. The angiogenic switch of human colon cancer occurs simultaneous to initiation of invasion. Oncology Reports 2003; 10: 9-13.

\section{Endereço para correspondência:} MARIO JUCÁ

Rua Adolfo Gustavo S/N - Lote 2 - Serraria Maceió - AL

57045-340 